\title{
ANALYSIS OF STRAIN LOCALIZATION IN POROUS MEDIA WITH TRANSVERSELY ISOTROPIC ELASTICITY UNDER UNDRAINED CONDITIONS
}

\author{
Y.Q. ZhANG, L.Z. WANG, M. PANG, L.F. FAN \\ College of Civil Engineering and Architecture, Zhejiang University, Hangzhou, China \\ e-mail:ppmmzju@163.com (M. Pang)
}

\begin{abstract}
The properties of strain localization for elastic-plastic porous media with transversely isotropic elasticity under undrained conditions are investigated. Under non-associated plasticity and tri-axial stress states, the conditions for strain localization of elastic-plastic porous media are derived, in which the effects of deviation from isotropic elasticity and pore fluid compressibility are included. Based on the Mohr-Coulomb yield criterion, the influences of the deviation from isotropic elasticity and pore fluid compressibility on the direction angle of localized band initiation and the corresponding critical hardening modulus for the case of plane strain are discussed. As a result, the properties of strain localization are dependent upon the deviation from isotropic elasticity and pore fluid compressibility. The deviation from isotropic elasticity and pore fluid compressibility has significant impacts on the direction angle of localized band initiation and the corresponding critical hardening modulus.
\end{abstract}

Keywords: porous medium, strain localization, transverse isotropic elasticity, undrained condition, pore fluid compressibility

\section{Introduction}

Strain localization of plastic flow into localized deformation bands is a typical feature of geomaterials such as rocks and soils undergoing non-homogeneous deformation. The onset of localized bands is a failure precursor as it signifies the initiation of an emerging localized failure mechanism. Strain localization is mathematically described as the inception of a discontinuous bifurcation in the form of a jump in the velocity gradient field within classical rate independent continuum theory. Analysis of strain localization may provide insight into the failure mechanism of engineering materials. Over the last decades, much attention has been drawn to the field of strain localization (Ottosen and Runesson, 1991; Bigoni and Loret, 1999; Rizzi and Loret, 1999; Zhang and Schrefler, 2001; Zhang et al., 2002; Longere and Dragon, 2007; Alyavuz and Gultop, 2009; Gao and Zhao, 2013). A suitable tool for delineating localization in solid mechanics is on the basis of strain rate discontinuity in continuum theory, and its basic principles were developed by Thomas (1961), Mandel (1962, 1964), and Rice (1976).

For a wide variety of constitutive models, conditions for the onset of strain localization were obtained by Ottosen and Runesson (1991), Neilsen and Schreyer (1993), Runesson et al. (1996), Zhang et al., 2002. In these studies, it was assumed that elasticity remained isotropic during the loading process. As a matter of fact, transversely isotropic materials are of primary interest in many engineering applications. Rudnicki (1977) proposed a transversely isotropic constitutive relation and elucidated properties of deformation localization of brittle rocks. Following the associated plastic flow rule, Rizzi and Loret (1997) presented the localization condition for an elastoplastic von Mises material with transversely isotropic elasticity under uniaxial tension. 
With associated plasticity and uniaxial tension, further developments concerning both transversely isotropic elasticity and plasticity were given by Loret and Rizzi (1997). Rudnicki (2002) derived the conditions for localized deformation in a transversely isotropic material under axisymmetric compression. For non-associated plasticity, Zhang et al. (2003) deduced the general description of properties of strain localization for elastoplastic materials with transversely isotropic elasticity subjected to tri-axial stress states. It is noted that these researches involves only the behaviour of one-phase transversely isotropic materials. As a matter of fact, the localization phenomena are relevant also for porous media with pores filled with a fluid. Based on the specific constitutive model developed by Rudnicki (1977) for transversely isotropic brittle rock, Zhang et al. (2005) analysed material instabilities of saturated multiphase porous media.

In this paper, the localization analysis is performed for elastic-plastic saturated porous media with transversely isotropic elasticity under undrained conditions. The general description of the properties of strain localization is deduced for porous media which follow non-associated plasticity and are subjected to tri-axial stress states. The effects of deviation from isotropic elasticity and pore fluid compressibility at plane strain are investigated. The porous body is assumed to undergo small deformations, and thus the nominal time rate is used instead of any objective rate measure.

\section{Elastic-plastic constitutive relations under undrained conditions}

For saturated porous media (e.g. soils), the deformation and strength under loading is not determined directly by the total stress but the effective stress. With compression being defined as positive, the effective stress can be expressed as

$$
\boldsymbol{\sigma}^{\prime}=\boldsymbol{\sigma}-p \mathbf{I}
$$

where $\boldsymbol{\sigma}$ is the total stress tensor, $\boldsymbol{\sigma}^{\prime}$ is the effective stress tensor, $p$ is the pore fluid pressure, and $\mathbf{I}$ is the second-order identity tensor. It is supposed that $\boldsymbol{\sigma}^{\prime}$ is responsible for deformation in the skeleton of the porous solid, whereas $p$ is responsible for compression of the pore fluid.

Under undrained conditions, it has the form

$$
\dot{p}=K^{f} \mathbf{I}: \dot{\varepsilon}
$$

where $\dot{\varepsilon}$ is the strain rate tensor, $K^{f}$ is the compression modulus of the pore fluid, and the symbol ' $:$ ' is the inner product with double index contraction. It should be pointed out that the effective stress principle defined by Eq. (2.1) is valid when the degree of saturation varies from 0.85 to 1.0. In this case, $K^{f}$ can be regarded as the bulk modulus of the two-phase mixture comprising liquid/air in the pores, and generally it may be two or three orders of magnitude smaller than that for the case of full saturation (Runesson et al., 1996).

Generally, the relationship between the total stress rate $\dot{\boldsymbol{\sigma}}$ and the strain rate $\dot{\boldsymbol{\varepsilon}}$ for plastic loading can be described by the incrementally linear relationship (Runesson et al., 1996)

$$
\dot{\boldsymbol{\sigma}}=\mathbf{D}_{u}: \dot{\varepsilon}
$$

where $\mathbf{D}_{u}$ is the total tangent stiffness tensor pertinent to the undrained condition, which is given by

$$
\mathbf{D}_{u}=\mathbf{D}^{\prime}+K^{f} \mathbf{I} \otimes \mathbf{I}
$$

where $\mathbf{D}^{\prime}$ denotes the effective elastic-plastic tangent stiffness tensor, and the symbol $\otimes$ designates the outer product of two tensors. Defining the yield function $F$ and the plastic potential $G$ in the effective stress space, we have

$$
\mathbf{D}^{\prime}=\mathbf{E}^{\prime}-\frac{1}{A^{\prime}}\left(\mathbf{E}^{\prime}: \mathbf{Q}^{\prime}\right) \otimes\left(\mathbf{P}^{\prime}: \mathbf{E}^{\prime}\right)
$$


where $\mathbf{P}^{\prime}$ and $\mathbf{Q}^{\prime}$ are the unit outward normals to the yield surface and the plastic potential, respectively, and they are defined as

$$
\mathbf{P}^{\prime}=\frac{\partial F}{\partial \boldsymbol{\sigma}^{\prime}} /\left\|\frac{\partial F}{\partial \boldsymbol{\sigma}^{\prime}}\right\| \quad \mathbf{Q}^{\prime}=\frac{\partial G}{\partial \boldsymbol{\sigma}^{\prime}} /\left\|\frac{\partial G}{\partial \boldsymbol{\sigma}^{\prime}}\right\|
$$

where $\|\cdot\|$ stands for a norm of a tensor. The positive parameter $A^{\prime}$ is defined as $A^{\prime}=H^{\prime}+\mathbf{P}^{\prime}$ : $\mathbf{E}^{\prime}: \mathbf{Q}^{\prime}$, where $H^{\prime}$ is the generalized plastic modulus and $\mathbf{E}^{\prime}$ is the effective elastic stiffness tensor.

For a transversely isotropic elastic material, it has the rotational symmetry property with reference to a certain axis. The plane perpendicular to this axis is called the basal plane while planes containing the axis of symmetry will be named the zonal planes. To better express material properties componentwise, the cartesian axes $\left(\mathbf{e}_{1}, \mathbf{e}_{2}, \mathbf{e}_{3}\right)$ are adopted, where $\mathbf{e}_{1}$ and $\mathbf{e}_{2}$ are two arbitrary orthogonal unit vectors of the basal plane, and the unit vector $\mathbf{e}_{3}$ denotes the axis of rotational symmetry. Thus, the stiffness $\mathbf{E}^{\prime}$ can take the form (Rizzi and Loret, 1997)

$$
\mathbf{E}^{\prime}=c_{1} \mathbf{I} \otimes \mathbf{I}+c_{2} \mathbf{I} \underline{\bar{\otimes}} \mathbf{I}+c_{3}(\mathbf{I} \otimes \mathbf{M}+\mathbf{M} \otimes \mathbf{I})+c_{4} \mathbf{M} \otimes \mathbf{M}+c_{5}(\mathbf{I} \underline{\bar{\otimes}} \mathbf{M}+\mathbf{M} \underline{\bar{\otimes}} \mathbf{I})
$$

where the tensor $\mathbf{M}=\mathbf{e}_{3} \otimes \mathbf{e}_{3}, c_{i}(i \in[1,5])$ are five material constants, and the symbol $\underline{\bar{Q}}$ denotes a symmetrized outer product, and it has $(\mathbf{M} \underline{\underline{Q}} \mathbf{I})_{i j k l}=\left(M_{i k} I_{j l}+M_{i l} I_{j k}\right) / 2$.

\section{Localization condition for non-associated plasticity}

It is known that the strain rate $\dot{\varepsilon}$ across the localized band is discontinuous. Assuming that $\dot{\varepsilon}^{i}$ and $\dot{\boldsymbol{\varepsilon}}^{o}$ denote the strain rates inside and outside the band, respectively, it follows from Eq. (2.3) that

$$
\dot{\boldsymbol{\sigma}}^{i}=\mathbf{D}_{u}^{i}: \dot{\varepsilon}^{i} \quad \dot{\boldsymbol{\sigma}}^{o}=\mathbf{D}_{u}^{o}: \dot{\boldsymbol{\varepsilon}}^{o}
$$

where $\dot{\boldsymbol{\sigma}}^{i}$ and $\dot{\boldsymbol{\sigma}}^{o}$ are the total stress rate inside and outside the localized band, respectively, and $\mathbf{D}_{u}^{i}$ and $\mathbf{D}_{u}^{o}$ denote the total tangential stiffness tensors inside and outside the band under undrained conditions, respectively. As mentioned by Zhang et al. (2003), the difference between the strain rates inside and outside the band is equal to $(\mathbf{m} \otimes \mathbf{n}+\mathbf{n} \otimes \mathbf{m}) / 2$ with the vector $\mathbf{m}$ the mode of discontinuity of the strain rate and $\mathbf{n}$ the unit normal vector of the band. The traction rate across the band must be unique, namely $\mathbf{n} \cdot\left(\dot{\boldsymbol{\sigma}}^{i}-\dot{\boldsymbol{\sigma}}^{o}\right)=0$ with symbol '. ' the inner product with single index contraction. Moreover, it can be regarded that $\mathbf{D}_{u}^{o}=\mathbf{D}_{u}^{i}=\mathbf{D}_{u}$ at the inception of strain localization. Thus, from Eq. (3.1), we have

$$
\mathbf{L} \cdot \mathbf{m}=0 \quad \text { or } \quad \operatorname{det}(\mathbf{L})=0
$$

which is the necessary condition for strain localization, and the total acoustic tensor under the undrained condition

$$
\mathbf{L}=\mathbf{n} \cdot \mathbf{D}_{u} \cdot \mathbf{n}=\mathbf{L}^{\prime}+K^{f} \mathbf{n} \otimes \mathbf{n}
$$

where $\mathbf{L}^{\prime}$ is the effective acoustic tensor, which can be expressed as

$$
\mathbf{L}^{\prime}=\mathbf{n} \cdot \mathbf{D}^{\prime} \cdot \mathbf{n}=\mathbf{L}_{e}^{\prime}-\frac{1}{A^{\prime}} \mathbf{a} \otimes \mathbf{b}
$$

where $\mathbf{L}_{e}^{\prime}$ denotes the effective elastic acoustic tensor, which is defined as

$$
\mathbf{L}_{e}^{\prime}=\mathbf{n} \cdot \mathbf{E}^{\prime} \cdot \mathbf{n}
$$


Additionally, the vectors $\mathbf{a}$ and $\mathbf{b}$ are formulated by

$$
\mathbf{a}=\mathbf{n} \cdot \mathbf{E}^{\prime}: \mathbf{Q}^{\prime} \quad \mathbf{b}=\mathbf{P}^{\prime}: \mathbf{E}^{\prime} \cdot \mathbf{n}
$$

Introduction of Eq. (2.7) into Eq. (3.5) yields (Zhang et al., 2003)

$$
\mathbf{L}_{e}^{\prime}=\alpha_{1} \mathbf{I}+\alpha_{2} \mathbf{n} \otimes \mathbf{n}+\alpha_{3}\left(\mathbf{e}_{3} \otimes \mathbf{n}+\mathbf{n} \otimes \mathbf{e}_{3}\right)+\alpha_{4} \mathbf{e}_{3} \otimes \mathbf{e}_{3}
$$

with

$$
\begin{array}{ll}
\alpha_{1}=\frac{c_{2}}{2}+\frac{c_{5}}{2}\left(\mathbf{e}_{3} \cdot \mathbf{n}\right)^{2} & \alpha_{2}=c_{1}+\frac{c_{2}}{2} \\
\alpha_{3}=\left(c_{3}+\frac{c_{5}}{2}\right)\left(\mathbf{e}_{3} \cdot \mathbf{n}\right) & \alpha_{4}=\left(\frac{c_{5}}{2}+c_{4}\right)\left(\mathbf{e}_{3} \cdot \mathbf{n}\right)^{2}
\end{array}
$$

Substitution of Eq. (2.7) into Eq. (3.6) leads to

$$
\mathbf{a}=\eta_{1} \mathbf{n}+\eta_{2} \mathbf{Q}^{\prime} \cdot \mathbf{n}+\eta_{3} \mathbf{e}_{3}+\eta_{4} \mathbf{Q}^{\prime} \cdot \mathbf{e}_{3} \quad \mathbf{b}=\chi_{1} \mathbf{n}+\chi_{2} \mathbf{P}^{\prime} \cdot \mathbf{n}+\chi_{3} \mathbf{e}_{3}+\chi_{4} \mathbf{P}^{\prime} \cdot \mathbf{e}_{3}
$$

with

$$
\begin{aligned}
& \eta_{1}=c_{1} \operatorname{tr} \mathbf{Q}^{\prime}+c_{3}\left(\mathbf{e}_{3} \cdot \mathbf{Q}^{\prime} \cdot \mathbf{e}_{3}\right) \quad \eta_{2}=c_{2} \\
& \eta_{3}=\left[c_{3} \operatorname{tr} \mathbf{Q}^{\prime}+c_{4}\left(\mathbf{e}_{3} \cdot \mathbf{Q}^{\prime} \cdot \mathbf{e}_{3}\right)\right]\left(\mathbf{e}_{3} \cdot \mathbf{n}\right)+c_{5}\left(\mathbf{n} \cdot \mathbf{Q}^{\prime} \cdot \mathbf{e}_{3}\right) \quad \eta_{4}=c_{5}\left(\mathbf{e}_{3} \cdot \mathbf{n}\right)
\end{aligned}
$$

and

$$
\begin{aligned}
& \chi_{1}=c_{1} \operatorname{tr} \mathbf{P}^{\prime}+c_{3}\left(\mathbf{e}_{3} \cdot \mathbf{P}^{\prime} \cdot \mathbf{e}_{3}\right) \quad \chi_{2}=c_{2} \\
& \chi_{3}=\left[c_{3} \operatorname{tr} \mathbf{P}^{\prime}+c_{4}\left(\mathbf{e}_{3} \cdot \mathbf{P}^{\prime} \cdot \mathbf{e}_{3}\right)\right]\left(\mathbf{e}_{3} \cdot \mathbf{n}\right)+c_{5}\left(\mathbf{n} \cdot \mathbf{P}^{\prime} \cdot \mathbf{e}_{3}\right) \quad \chi_{4}=c_{5}\left(\mathbf{e}_{3} \cdot \mathbf{n}\right)
\end{aligned}
$$

where the symbol "tr" denotes the trace operator of tensors.

When the tensor $\mathbf{L}$ is singular, the corresponding hardening modulus can be obtained by

$$
H^{\prime}=\mathbf{a} \cdot \mathbf{R}_{e}^{\prime} \cdot \mathbf{b}-\psi \frac{\left(\mathbf{a} \cdot \mathbf{R}_{e}^{\prime} \cdot \mathbf{n}\right)\left(\mathbf{b} \cdot \mathbf{R}_{e}^{\prime} \cdot \mathbf{n}\right)}{\mathbf{n} \cdot \mathbf{R}_{e}^{\prime} \cdot \mathbf{n}}-\mathbf{P}^{\prime}: \mathbf{E}^{\prime}: \mathbf{Q}^{\prime}=h_{n}-h_{E}
$$

where $\psi=K^{f} \mathbf{n} \cdot \mathbf{R}_{e}^{\prime} \cdot \mathbf{n} /\left(1+K^{f} \mathbf{n} \cdot \mathbf{R}_{e}^{\prime} \cdot \mathbf{n}\right)$, and

$$
h_{n}=\mathbf{a} \cdot \mathbf{R}_{e}^{\prime} \cdot \mathbf{b}-\psi \frac{\left(\mathbf{a} \cdot \mathbf{R}_{e}^{\prime} \cdot \mathbf{n}\right)\left(\mathbf{b} \cdot \mathbf{R}_{e}^{\prime} \cdot \mathbf{n}\right)}{\mathbf{n} \cdot \mathbf{R}_{e}^{\prime} \cdot \mathbf{n}} \quad h_{E}=\mathbf{P}^{\prime}: \mathbf{E}^{\prime}: \mathbf{Q}^{\prime}
$$

where $\mathbf{R}_{e}^{\prime}$ is the inverse of the effective elastic acoustic tensor $\mathbf{L}_{e}^{\prime}$, which is expressed as (Zhang et al., 2003)

$$
\mathbf{R}_{e}^{\prime}=\beta_{1} \mathbf{I}+\beta_{2} \mathbf{n} \otimes \mathbf{n}+\beta_{3}\left(\mathbf{e}_{3} \otimes \mathbf{n}+\mathbf{n} \otimes \mathbf{e}_{3}\right)+\beta_{4} \mathbf{e}_{3} \otimes \mathbf{e}_{3}
$$

where

$$
\begin{aligned}
& \beta_{1}=\frac{1}{\alpha_{1}} \quad \beta_{2}=\frac{1}{\alpha_{1} \Delta}\left[-\alpha_{2}\left(\alpha_{1}+\alpha_{4}\right)+\alpha_{3}^{2}\right] \\
& \beta_{3}=\frac{1}{\alpha_{1} \Delta}\left[-\alpha_{1} \alpha_{3}+\left(\alpha_{2} \alpha_{4}-\alpha_{3}^{2}\right)\left(\mathbf{e}_{3} \cdot \mathbf{n}\right)\right] \quad \beta_{4}=\frac{1}{\alpha_{1} \Delta}\left[-\alpha_{4}\left(\alpha_{1}+\alpha_{2}\right)+\alpha_{3}^{2}\right]
\end{aligned}
$$

with

$$
\begin{aligned}
\Delta= & \frac{1}{2}\left(c_{1}+c_{2}\right)\left(c_{2}+c_{5}\right)+\left[\left(c_{3}+c_{5}\right)\left(c_{2}-c_{3}\right)+c_{4}\left(c_{1}+c_{2}\right)\right]\left(\mathbf{e}_{3} \cdot \mathbf{n}\right)^{2} \\
& +\frac{1}{2}\left[2 c_{3}\left(c_{3}+2 c_{5}\right)-c_{4}\left(2 c_{1}+c_{2}\right)+c_{5}\left(c_{4}+2 c_{5}\right)\right]\left(\mathbf{e}_{3} \cdot \mathbf{n}\right)^{4}
\end{aligned}
$$


Substituting Eq. (2.7) into Eq. (3.13), we have

$$
\begin{aligned}
h_{E} & =c_{1}\left(\operatorname{tr} \mathbf{P}^{\prime}\right)\left(\operatorname{tr} \mathbf{Q}^{\prime}\right)+c_{2} \mathbf{P}^{\prime}: \mathbf{Q}^{\prime}+c_{3}\left[\left(\mathbf{e}_{3} \cdot \mathbf{Q}^{\prime} \cdot \mathbf{e}_{3}\right) \operatorname{tr} \mathbf{P}^{\prime}+\left(\mathbf{e}_{3} \cdot \mathbf{P}^{\prime} \cdot \mathbf{e}_{3}\right) \operatorname{tr} \mathbf{Q}^{\prime}\right] \\
& +c_{4}\left(\mathbf{e}_{3} \cdot \mathbf{P}^{\prime} \cdot \mathbf{e}_{3}\right)\left(\mathbf{e}_{3} \cdot \mathbf{Q}^{\prime} \cdot \mathbf{e}_{3}\right)+2 c_{5}\left(\mathbf{e}_{3} \cdot\left(\mathbf{P}^{\prime} \cdot \mathbf{Q}^{\prime}\right) \cdot \mathbf{e}_{3}\right)
\end{aligned}
$$

As strain localization may occur along a surface of normal $n$ when the elastoplastic acoustic tensor gets first singular in that direction, the most critical directions $\mathbf{n}$ will be those corresponding to the largest hardening modulus which makes the elastoplastic acoustic tensor singular. Thus, from Eq. (3.12), we have

$$
H_{c r}^{\prime}=\max _{\mathbf{n}, \mathbf{n} \cdot \mathbf{n}=1} H^{\prime}(\mathbf{n})=\max _{\mathbf{n}, \mathbf{n} \cdot \mathbf{n}=1}\left(h_{\mathbf{n}}\right)-h_{E}
$$

It is noted from Eq. (2.7) that the material constants $c_{3}, c_{4}$, and $c_{5}$ can be interpreted as measures of the deviation from isotropic elasticity. When $c_{3}=c_{4}=c_{5}=0$, Eq. (2.7) reduces to the case of isotropic elasticity. Assuming that $\lambda$ and $\mu$ denote the Lamé coefficients of an isotropic reference elastic material with positive definite elastic stiffness, it has $3 \lambda+2 \mu>0$ and $\mu>0$ (Zhang et al., 2003). Rizzi and Loret (1997) carried out numerical simulations on three prototypes of anisotropic materials due to difficulties of theoretical derivations. It was found to include most of the qualitative features common to other simulations for the case of $c_{1}=\lambda$, $c_{2}=2 \mu, c_{3}=c_{5}=0$, and $c_{4} \neq 0$. As a consequence, the analysis in this study is confined to this case for simplicity in the mathematical derivations. For this case, we have (Zhang et al., 2003)

$$
\begin{array}{ll}
E_{L}=\frac{\mu(3 \lambda+2 \mu)+(\lambda+\mu) c_{4}}{\lambda+\mu} \quad E_{T}=\frac{4 \mu\left[\mu(3 \lambda+2 \mu)+c_{4}(\lambda+\mu)\right]}{4 \mu \lambda+\mu+c_{4}(\lambda+2 \mu)} \\
G_{l}=G_{T}=\mu \quad \nu_{L}=\frac{\lambda}{2(\lambda+\mu}
\end{array}
$$

where $E_{L}$ and $E_{T}$, respectively, denote the longitudinal (or axial) elastic modulus and the transverse (or cross-axial) elastic modulus, $G_{L}$ and $G_{T}$, respectively, denote the longitudinal (or zonal) shear modulus and the transverse (or basal) shear modulus, and $\nu_{L}$ the longitudinal Poisson ratio (representing the contraction in the longitudinal direction due to an imposed traction in the basal plane).

Corresponding to the Loss of Positive Definiteness (LPD) of the elastic stiffness, there is a lower bound for the admissible values of the elastic parameter $c_{4}$, which is herein denoted by $c_{4}^{L P D}$ and given by

$$
c_{4}>c_{4}^{L P D}=-\frac{\mu(3 \lambda+2 \mu)}{\lambda+\mu}=-E
$$

where $E$ is Young's modulus corresponding to the assumed Lamé constants.

In view of the above simplification, it follows from Eq. (3.13) that

$$
h_{n}=U_{1}+U_{2}-\frac{K^{f} U_{3} U_{4}}{1+K^{f} U_{5}}
$$

with

$$
\begin{aligned}
U_{1} & =\left[2 c_{1}^{2} \beta_{3} P_{v}^{\prime} Q_{v}^{\prime}+c_{1} c_{4} \beta_{3}\left(P_{v}^{\prime} Q_{33}^{\prime}+Q_{v}^{\prime} P_{33}^{\prime}\right)\right] n_{3}+\left[c_{2} c_{4}\left(\beta_{1}+\beta_{4}\right)\left(Q_{33}^{\prime} P_{3 j}^{\prime} n_{j}+P_{33}^{\prime} Q_{3 j}^{\prime} n_{j}\right)\right. \\
& \left.+c_{1} c_{2} \beta_{4}\left(Q_{v}^{\prime} P_{3 j}^{\prime} n_{j}+P_{v}^{\prime} Q_{3 j}^{\prime} n_{j}\right)\right] n_{3}+\left[c_{1} c_{2} \beta_{3}\left(P_{v}^{\prime}+Q_{v}^{\prime}\right)+c_{2} c_{4} \beta_{3}\left(P_{33}^{\prime}+Q_{33}^{\prime}\right)\right]\left(n_{i} Q_{i j}^{\prime} n_{j}\right) n_{3} \\
& +\left[c_{1}^{2} \beta_{4} P_{v}^{\prime} Q_{v}^{\prime}+c_{1} c_{4}\left(\beta_{2}+\beta_{4}+\beta_{1}\right)\left(P_{v}^{\prime} Q_{33}^{\prime}+Q_{v}^{\prime} P_{33}^{\prime}\right)+c_{4}^{2}\left(\beta_{1}+\beta_{4}\right) P_{33}^{\prime} Q_{33}^{\prime}\right] n_{3}^{2} \\
& +c_{2} c_{4} \beta_{2}\left(Q_{33}^{\prime} n_{i} P_{i j}^{\prime} n_{j}+P_{33}^{\prime} n_{i} Q_{i j}^{\prime} n_{j}\right) n_{3}^{2}+c_{2} c_{4} \beta_{3}\left(P_{33}^{\prime} Q_{3 j}^{\prime} n_{j}+Q_{33}^{\prime} P_{3 j}^{\prime} n_{j}\right) n_{3}^{2}
\end{aligned}
$$




$$
\begin{aligned}
U_{2} & =c_{4}^{2} \beta_{2} Q_{33}^{\prime} P_{33}^{\prime} n_{3}^{4}+c_{2}^{2} \beta_{4} P_{3 j}^{\prime} n_{j} Q_{3 k}^{\prime} n_{k}+c_{1} c_{2} \beta_{3}\left(Q_{3 j}^{\prime} n_{j} P_{v}^{\prime}+P_{3 j}^{\prime} n_{j} Q_{v}^{\prime}\right) \\
& +c_{2}^{2} \beta_{2} n_{i} Q_{i j}^{\prime} n_{j} n_{k} P_{k l}^{\prime} n_{l}+c_{2}^{2} \beta_{1} n_{i} P_{i k}^{\prime} Q_{k j}^{\prime} n_{j}+c_{2}^{2} \beta_{3}\left(P_{3 j}^{\prime} n_{j} n_{k} Q_{k l}^{\prime} n_{l}+Q_{3 j}^{\prime} n_{j} n_{k} P_{k l}^{\prime} n_{l}\right) \\
& +c_{1}^{2}\left(\beta_{1}+\beta_{2}\right) P_{v}^{\prime} Q_{v}^{\prime}+c_{1} c_{2}\left(\beta_{1}+\beta_{2}\right)\left(P_{v}^{\prime} n_{i} Q_{i j}^{\prime} n_{j}+Q_{v}^{\prime} n_{i} P_{i j}^{\prime} n_{j}\right) \\
& +\left[c_{1} c_{4} \beta_{3}\left(Q_{v}^{\prime} P_{33}^{\prime}+P_{v}^{\prime} Q_{33}^{\prime}\right)+2 c_{4}^{2} \beta_{3} Q_{33}^{\prime} P_{33}^{\prime}\right] n_{3}^{3} \\
U_{3} & =c_{1} Q_{v}^{\prime}\left(\beta_{1}+\beta_{2}+2 \beta_{3} n_{3}+\beta_{4} n_{3}^{2}\right)+c_{2} n_{i} Q_{i j}^{\prime} n_{j}\left(\beta_{1}+\beta_{2}+\beta_{3} n_{3}\right) \\
& +c_{2} Q_{3 j}^{\prime} n_{j}\left(\beta_{3}+\beta_{4} n_{3}\right)+c_{4} Q_{33}^{\prime} n_{3}\left[\beta_{3}+\left(\beta_{1}+\beta_{2}+\beta_{4}\right) n_{3}+\beta_{3} n_{3}^{2}\right] \\
U_{4} & =c_{1} P_{v}^{\prime}\left(\beta_{1}+\beta_{2}+2 \beta_{3} n_{3}+\beta_{4} n_{3}^{2}\right)+c_{2} n_{i} P_{i j}^{\prime} n_{j}\left(\beta_{1}+\beta_{2}+\beta_{3} n_{3}\right)+c_{2} P_{3 j}^{\prime} n_{j}\left(\beta_{3}+\beta_{4} n_{3}\right) \\
& +c_{4} P_{33}^{\prime} n_{3}\left[\beta_{3}+\left(\beta_{1}+\beta_{2}+\beta_{4}\right) n_{3}+\beta_{3} n_{3}^{2}\right] \\
U_{5} & =\beta_{1}+\beta_{2}+2 \beta_{3} n_{3}+\beta_{4} n_{3}^{2}
\end{aligned}
$$

and

$$
h_{E}=c_{1} P_{v}^{\prime} Q_{v}^{\prime}+c_{2} P_{i j}^{\prime} Q_{i j}^{\prime}+c_{4} P_{33}^{\prime} Q_{33}^{\prime}
$$

where the summation convention is adopted for Latin indices, $P_{v}^{\prime}=\operatorname{tr} \mathbf{P}^{\prime}$, and $Q_{v}^{\prime}=\operatorname{tr} \mathbf{Q}^{\prime}$.

When the modulus $K^{f}=0$, Eq. (3.20) can be reduced to the solution of the single-phase solid obtained by Zhang et al. (2003). When the parameter $c_{4}=0$, Eq. (3.20) can be simplified to the solution given by Runesson et al. (1996).

\section{Properties of the localized band at plane strain}

In the case of plane strain, assuming the stress principal directions are consistent with the symmetrical axes of material and the components $n_{1}$ and $n_{3}$ are located in the plane of interest, for associated plasticity Eqs. (3.20) and (3.21) can be reduced to

$$
h_{n}=V_{1}+V_{2}-\frac{K^{f}\left(V_{3}\right)^{2}}{1+K^{f} V_{4}}
$$

with

$$
\begin{aligned}
V_{1}= & c_{1} P_{v}^{\prime}\left(\beta_{1}+\beta_{2}\right)\left[c_{1} P_{v}^{\prime}+2 c_{2}\left(P_{1}^{\prime} n_{1}^{2}+P_{3}^{\prime} n_{3}^{2}\right)\right] \\
& +c_{2}^{2}\left(P_{1}^{\prime 2} n_{1}^{2}+P_{3}^{\prime 2} n_{3}^{2}\right)\left[\beta_{1}+\beta_{2}\left(P_{1}^{\prime 2} n_{1}^{2}+P_{3}^{\prime 2} n_{3}^{2}\right)\right] \\
& +2 \beta_{3}\left[c_{1} P_{v}^{\prime}+\left(c_{4}+c_{2}\right) P_{3}^{\prime}\left[c_{1} P_{v}^{\prime}+c_{2}\left(P_{1}^{\prime} n_{1}^{2}+P_{3}^{\prime} n_{3}^{2}\right)\right] n_{3}\right. \\
V_{2}= & {\left[\left(2 c_{2} c_{4}+c_{4}^{2}\right)\left(\beta_{1}+\beta_{4}\right) P_{3}^{\prime 2}+c_{1} \beta_{4} P_{v}^{\prime}\left(2 c_{2} P_{3}^{\prime}+c_{1} P_{v}^{\prime}\right)\right.} \\
& \left.+2 c_{1} c_{4}\left(\beta_{1}+\beta_{2}+\beta_{4}\right) P_{3}^{\prime} P_{v}^{\prime}+c_{2}^{2} \beta_{4} P_{3}^{\prime 2}\right] n_{3}^{2}+2 c_{2} c_{4} \beta_{2} P_{3}^{\prime}\left(P_{1}^{\prime} n_{1}^{2}+P_{3}^{\prime} n_{3}^{2}\right) n_{3}^{2} \\
& +2 c_{4} \beta_{3} P_{3}^{\prime}\left(c_{2} P_{3}^{\prime}+c_{1} P_{v}^{\prime}+c_{4} P_{3}^{\prime}\right) n_{3}^{3}+c_{4}^{2} \beta_{2} P_{3}^{\prime 2} n_{3}^{4} \\
V_{3}= & c_{1} P_{v}^{\prime}\left(\beta_{1}+\beta_{2}+2 \beta_{3} n_{3}+\beta_{4} n_{3}^{2}\right)+c_{2}\left(\beta_{1}+\beta_{2}+\beta_{3} n_{3}\right)\left(P_{1}^{\prime} n_{1}^{2}+P_{3}^{\prime} n_{3}^{2}\right) \\
& +c_{2}\left(\beta_{3}+\beta_{4} n_{3}\right) P_{3}^{\prime} n_{3}+c_{4} P_{3}^{\prime} n_{3}\left[\beta_{3}+\left(\beta_{1}+\beta_{2}+\beta_{4}\right) n_{3}+\beta_{3} n_{3}^{2}\right] \\
V_{4}= & \beta_{1}+\beta_{2}+2 \beta_{3} n_{3}+\beta_{4} n_{3}^{2}
\end{aligned}
$$

and

$$
h_{E}=c_{1} P_{v}^{\prime 2}+c_{2}\left(P_{1}^{\prime 2}+P_{2}^{\prime 2}+P_{3}^{\prime 2}\right)+c_{4} P_{3}^{\prime 2}
$$

It should be mentioned that for the present case of plane strain we have

$$
n_{1}^{2}+n_{3}^{2}=1 \quad n_{1}^{2}=1-n_{3}^{2}
$$


Combination of Eqs. (3.8), (3.15), (3.16), (4.1) and (4.3) yields

$$
h_{\mathbf{n}}=\frac{1}{u^{2} \Delta}\left[u^{2}\left(r_{4} n_{3}^{4}+r_{2} n_{3}^{2}+r_{0}\right)-\frac{K^{f}\left(s_{5} n_{3}^{5}+s_{4} n_{3}^{4}+s_{2} n_{3}^{2}+s_{0}\right)^{2}}{\Delta+K^{f}\left(u+c_{4} n_{3}^{2}-c_{4} n_{3}^{4}\right)}\right]
$$

where

$$
\begin{aligned}
r_{4}= & -P_{3}^{\prime 2}(\lambda+\mu) c_{4}^{2}+\left[4 \mu P_{1}^{\prime} P_{3}^{\prime}(\lambda+\mu)-4 \mu P_{3}^{\prime 2}(\lambda+\mu)-4 \mu^{2} P_{1}^{\prime 2}\right. \\
& \left.-4 \lambda \mu P_{1}^{\prime} P_{v}^{\prime}-\lambda^{2} P_{v}^{\prime 2}\right] c_{4}-4 \mu^{2}\left(P_{3}^{\prime}-P_{1}^{\prime}\right)^{2}(\lambda+\mu) \\
r_{2}= & (\lambda+2 \mu) P_{3}^{\prime 2} c_{4}^{2}+\left[4 \mu P_{3}^{\prime 2}(\lambda+2 \mu)-4 \mu P_{1}^{\prime} P_{3}^{\prime}(\lambda+\mu)+4 \mu^{2} P_{1}^{\prime 2}\right. \\
& \left.+\lambda^{2} P_{v}^{\prime 2}+4 \lambda \mu P_{v}^{\prime} P_{1}^{\prime}+2 \lambda \mu P_{3}^{\prime} P_{v}^{\prime}\right] c_{4}+4 \lambda \mu^{2} P_{v}^{\prime}\left(P_{3}^{\prime}-P_{1}^{\prime}\right) \\
& +4 \mu^{2}\left(P_{3}^{\prime}-P_{1}^{\prime}\right)\left[\left(P_{3}^{\prime}+P_{1}^{\prime}\right)(\lambda+2 \mu)-2 P_{1}^{\prime}(\lambda+\mu)\right] \\
r_{0}= & 4 \mu^{3} P_{1}^{\prime 2}+\lambda^{2} \mu P_{v}^{\prime 2}+4 \lambda \mu^{2} P_{1}^{\prime} P_{v}^{\prime}
\end{aligned}
$$

and

$$
\begin{aligned}
& s_{5}=P_{3}^{\prime}(\lambda+\mu) c_{4}^{2} \quad s_{4}=c_{4}\left[P_{3}^{\prime}(\lambda+2 \mu) c_{4}-\lambda \mu P_{v}^{\prime}-2 \mu^{2} P_{1}\right] \\
& s_{2}=\mu c_{4}\left(\lambda P_{v}^{\prime}+2 \mu P_{1}^{\prime}\right)+2 \mu^{3}\left(P_{3}^{\prime}-P_{1}^{\prime}\right)+\mu^{2} c_{4} P_{3}^{\prime} \quad s_{0}=\mu^{2}\left(\lambda P_{v}^{\prime}+2 \mu P_{1}^{\prime}\right)
\end{aligned}
$$

Additionally, we have

$$
\Delta=\mu(\lambda+2 \mu)+c_{4}(\lambda+2 \mu) n_{3}^{2}-c_{4}(\lambda+\mu) n_{3}^{4}
$$

It is seen from Eq. (3.18) that the critical hardening modulus $H_{c r}^{\prime}$ corresponding to the initiation of strain localization is defined as the constrained maximization of $H^{\prime}$ over all possible localized band directions $\mathbf{n}$ for a given state. Assuming that the maximum value of $H^{\prime}$ is reached when $n_{3}=n_{3}^{c r}$, it has $\tan ^{2} \theta=\left(n_{3}^{c r}\right)^{2} /\left[1-\left(n_{3}^{c r}\right)^{2}\right]$, where $\theta$ denotes the angle in the $\mathbf{e}_{1}-\mathbf{e}_{3}$ plane from the $\mathbf{e}_{1}$-axis to the normal vector $\left(n_{1}, n_{3}\right)$ as shown in Fig. 1 . Then substituting $n_{3}^{c r}$ into Eq. (4.4) and combining Eq. (4.2), the corresponding maximum value of the hardening modulus $H_{c r}^{\prime}$ can be obtained.

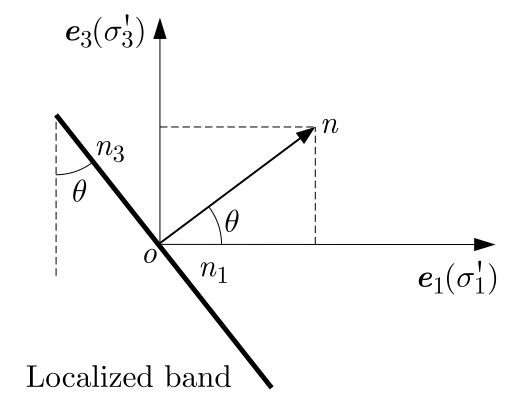

Fig. 1. Geometric relationship among the anisotropy axes, stress principal axes and the localized band direction at plane strain

\section{Numerical results and discussions}

In this Section, the properties of strain localization at strain plane is investigated based on the Mohr-Coulomb yield criterion. The Mohr-Coulomb yield criterion can be defined by

$$
F=\frac{1}{2}\left(\sigma_{\mathrm{I}}^{\prime}-\sigma_{\mathrm{III}}^{\prime}\right)+\frac{1}{2}\left(\sigma_{\mathrm{I}}^{\prime}+\sigma_{\mathrm{III}}^{\prime}\right) \sin \varphi-c=0
$$

where $\sigma_{\mathrm{I}}^{\prime} \geqslant \sigma_{\mathrm{II}}^{\prime} \geqslant \sigma_{\mathrm{III}}^{\prime}$ are the effective principal stresses (which are taken positive in compression), $\varphi$ is the angle of internal friction, and $c$ is a cohesion intercept. 
As the direction of the effective principal stress $\sigma_{3}^{\prime}$ is assumed to be consistent with the cartesian axe $\mathbf{e}_{3}$, there exist two situations for the in-plane effective principal stresses, namely $\sigma_{1}^{\prime} \geqslant \sigma_{3}^{\prime}$ and $\sigma_{3}^{\prime} \geqslant \sigma_{1}^{\prime}$. For the situation $\sigma_{1}^{\prime} \geqslant \sigma_{3}^{\prime}$, there are three cases depending on the magnitude of the out-of-plane stress $\sigma_{2}^{\prime}$.

\section{Case A}

When $\sigma_{1}^{\prime} \geqslant \sigma_{2}^{\prime} \geqslant \sigma_{3}^{\prime}$, it has $\sigma_{\mathrm{I}}^{\prime}=\sigma_{1}$ and $\sigma_{\mathrm{III}}=\sigma_{3}^{\prime}$, and from Eqs. (2.6) and (5.1), we obtain

$$
P_{1}^{\prime}=\frac{\sqrt{2}}{2} \frac{1+\sin \varphi}{\sqrt{1+\sin ^{2} \varphi}} \quad P_{2}^{\prime}=0 \quad P_{3}^{\prime}=\frac{-\sqrt{2}}{2} \frac{1-\sin \varphi}{\sqrt{1+\sin ^{2} \varphi}}
$$

and

$$
P_{v}^{\prime}=\frac{\sqrt{2} \sin \varphi}{\sqrt{1+\sin ^{2} \varphi}}
$$

Substituting Eqs. (5.2) and (5.3) into Eqs. (4.2) and (4.4) and combining Eq. (3.18), the critical hardening modulus and the band direction angle at the onset of strain localization can be determined. Suppose $\lambda / \mu=1$, we know from Eq. (3.19) that $c_{4} / \mu>-2.5$. In addition, for soils the magnitude of the ratio $K^{f} /(2 \mu)$ may range from $10^{-1}$ to $10^{3}$ in practice, which represent extreme states for moduli for partial and full liquid saturation, respectively. Thus, with $\varphi=10^{\circ}$, the influences of the parameters $c_{4}$ and $K^{f}$ on the angle $\theta$ and the critical hardening modulus $H_{c r}^{\prime}$ are shown in Figs. 2a and 2b, respectively. As can be seen from Fig. 2a, the angle $\theta$ first increases with the increase of the parameter $c_{4}$, and then decreases with its further increase. The influence of the modulus $K^{f}$ on the angle $\theta$ is related to the magnitude of the parameter $c_{4}$. It can be observed from Fig. $2 \mathrm{~b}$ that at first the critical hardening modulus $H_{c r}^{\prime}$ becomes larger with the increase of the parameter $c_{4}$, but it becomes smaller with the further increase of the parameter $c_{4}$ when $-2.5<c_{4} / \mu \leqslant 0$ or $c_{4} \geqslant 0$. In addition, the critical hardening modulus $H_{c r}^{\prime}$ generally decreases with the increase of the modulus $K^{f}$.

(a)

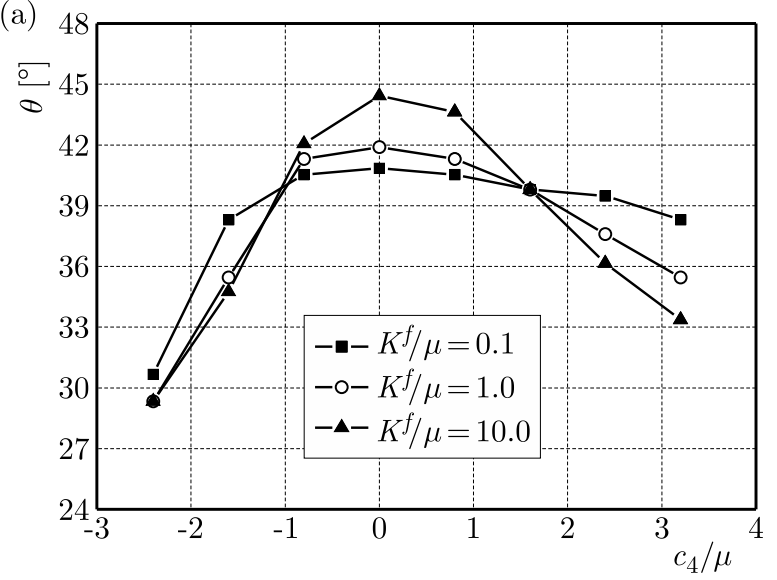

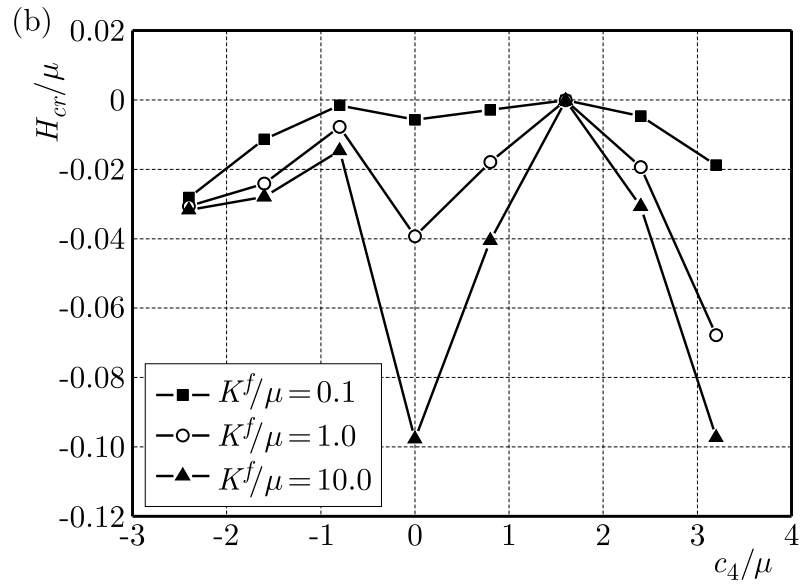

Fig. 2. Variation of the band direction angle $\theta$ (a) and the critical hardening modulus $H_{c r}^{\prime}$ (b) with the parameter $c_{4}$ for various $K^{f}$ in the case $\sigma_{1}^{\prime} \geqslant \sigma_{2}^{\prime} \geqslant \sigma_{3}^{\prime}$

\section{Case B}

When $\sigma_{1}^{\prime} \geqslant \sigma_{3}^{\prime} \geqslant \sigma_{2}^{\prime}$, it has $\sigma_{\mathrm{I}}^{\prime}=\sigma_{1}^{\prime}$ and $\sigma_{\mathrm{III}}^{\prime}=\sigma_{2}^{\prime}$. Then it follows from Eqs. (2.6) and (5.1) that

$$
P_{1}^{\prime}=\frac{\sqrt{2}}{2} \frac{1+\sin \varphi}{\sqrt{1+\sin ^{2} \varphi}} \quad P_{2}^{\prime}=\frac{-\sqrt{2}}{2} \frac{1-\sin \varphi}{\sqrt{1+\sin ^{2} \varphi}} \quad P_{3}^{\prime}=0
$$

and 


$$
P_{v}^{\prime}=\frac{\sqrt{2} \sin \varphi}{\sqrt{1+\sin ^{2} \varphi}}
$$

Thus, assuming $\lambda / \mu=1$ and $\varphi=10^{\circ}$, the impacts of the parameters $c_{4}$ and $K^{f}$ on the band angle $\theta$ and the critical hardening modulus $H_{c r}^{\prime}$ are illustrated in Figs. 3a and 3b, respectively. As can be seen, the direction angle of localized band initiation and the corresponding critical hardening modulus are significantly dependent upon the deviation from isotropic elasticity and pore fluid compressibility. For the effect of deviation from isotropic elasticity, it is shown that a larger value of the parameter $c_{4}$ leads to smaller values of the band angle $\theta$ and the critical hardening modulus $H_{c r}^{\prime}$. As for the effect of pore fluid compressibility, it is indicated that a larger value of the modulus $K^{f}$ results in a larger value of the band angle $\theta$ for the vast majority of cases and a smaller value of the critical hardening modulus $H_{c r}^{\prime}$.

(a)

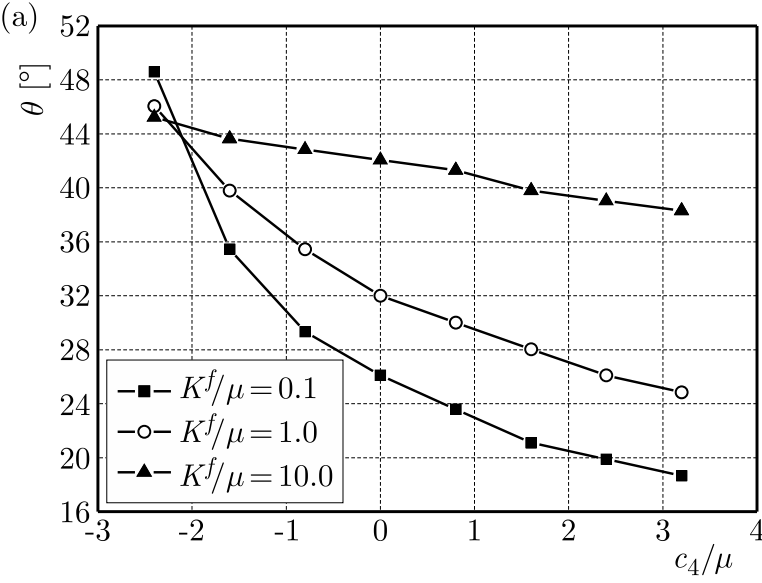

(b)

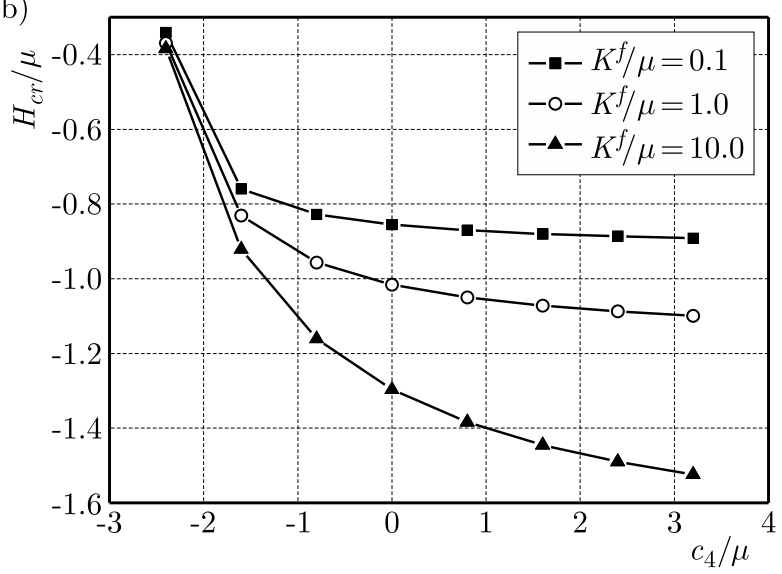

Fig. 3. Variation of the band direction angle $\theta$ (a) and the critical hardening modulus $H_{c r}^{\prime}$ (b) with the parameter $c_{4}$ for various $K^{f}$ in the case $\sigma_{1}^{\prime} \geqslant \sigma_{3}^{\prime} \geqslant \sigma_{2}^{\prime}$

\section{Case C}

When $\sigma_{2}^{\prime} \geqslant \sigma_{1}^{\prime} \geqslant \sigma_{3}^{\prime}$, it has $\sigma_{\mathrm{I}}^{\prime}=\sigma_{2}^{\prime}, \sigma_{\mathrm{III}}^{\prime}=\sigma_{3}^{\prime}$. Hence, combination of Eqs. (2.6) and (5.1) yields

$$
P_{1}^{\prime}=0 \quad P_{2}^{\prime}=\frac{\sqrt{2}}{2} \frac{1+\sin \varphi}{\sqrt{1+\sin ^{2} \varphi}} \quad P_{3}^{\prime}=\frac{-\sqrt{2}}{2} \frac{1-\sin \varphi}{\sqrt{1+\sin ^{2} \varphi}}
$$

and

$$
P_{v}^{\prime}=\frac{\sqrt{2} \sin \varphi}{\sqrt{1+\sin ^{2} \varphi}}
$$

In this case, with $\lambda / \mu=1$ and $\varphi=10^{\circ}$, the dependences of the band direction angle at the inception of strain localization and the corresponding critical hardening modulus on the deviation from isotropic elasticity and pore fluid compressibility are indicated in Figs. 4a and $4 \mathrm{~b}$, respectively. It can be observed that the band direction angle $\theta$ and the critical hardening modulus $H_{c r}^{\prime}$ get smaller with an increase in the value of modulus $K^{f}$. However, it should be noted that the influence of pore fluid compressibility on the critical hardening modulus is very limited when $c_{4} / \mu$ ranges between -2.5 and -1 . On the other hand, it is found that the effects of the deviation from isotropic elasticity on the band direction angle and the corresponding critical hardening modulus are obvious. When $c_{4} \geqslant 0$, the band direction angle $\theta$ diminishes with an increase in the parameter $c_{4}$. When $c_{4} \leqslant 0$, the band direction angle $\theta$ becomes larger with the 
(a)

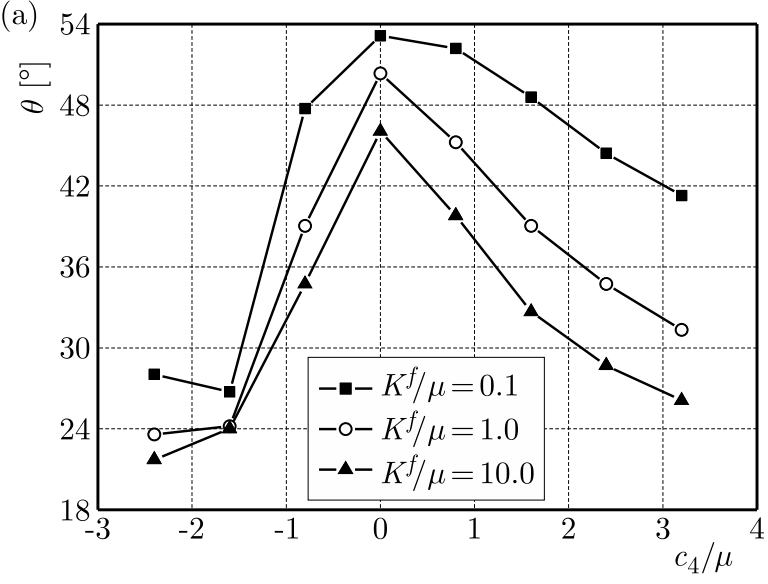

(b)

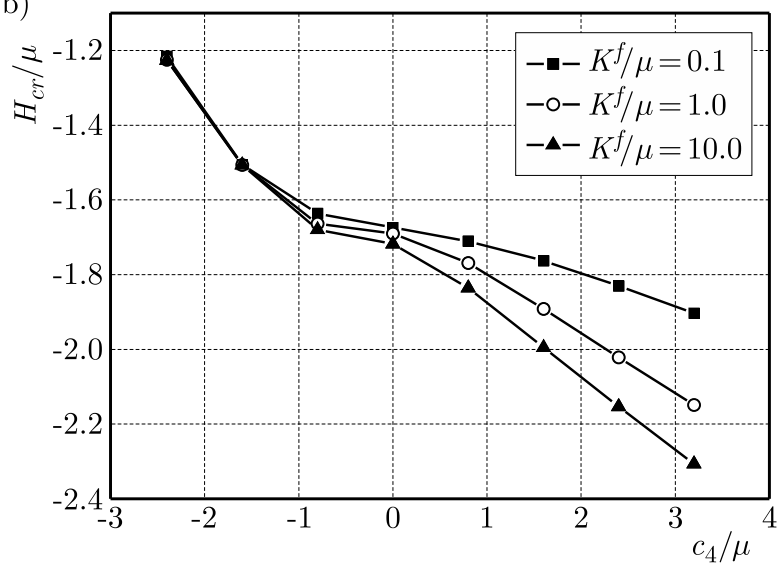

Fig. 4. Variation of the band direction angle $\theta$ (a) and the critical hardening modulus $H_{c r}^{\prime}(\mathrm{b})$ with the parameter $c_{4}$ for various $K^{f}$ in the case $\sigma_{2}^{\prime} \geqslant \sigma_{1}^{\prime} \geqslant \sigma_{3}^{\prime}$

increasing parameter $c_{4}$ in the cases of $K^{f} / \mu=1$ and $K^{f} / \mu=10$, whereas it first decreases and then increases with the diminishment of the parameter $c_{4}$ in the case of $K^{f} / \mu=0.1$. Additionally, the critical hardening modulus $H_{c r}^{\prime}$ becomes smaller with an increase in the parameter $c_{4}$.

For the situation $\sigma_{3}^{\prime} \geqslant \sigma_{1}^{\prime}$, there also exist three cases depending on the magnitude of the out-of-plane stress $\sigma_{2}^{\prime}$, which are defined by $\sigma_{3} \geqslant \sigma_{2} \geqslant \sigma_{1}, \sigma_{3} \geqslant \sigma_{1} \geqslant \sigma_{2}$, and $\sigma_{2} \geqslant \sigma_{3} \geqslant \sigma_{1}$, respectively. Similar to those cases for the situation $\sigma_{1}^{\prime} \geqslant \sigma_{3}^{\prime}$, with $\lambda / \mu=1$ and $\varphi=10^{\circ}$ the influences of the deviation from isotropic elasticity and pore fluid compressibility on the band direction angle at the initiation of strain localization and the corresponding critical hardening modulus are calculated and shown in Figs. 5-7. Among these figures, Figs. 5a and 5b are for the case $\sigma_{3} \geqslant \sigma_{2} \geqslant \sigma_{1}$, Figs. $6 \mathrm{a}$ and $6 \mathrm{~b}$ are for the case $\sigma_{3} \geqslant \sigma_{1} \geqslant \sigma_{2}$, and Figs. 7a and $7 \mathrm{~b}$ are for the case $\sigma_{2} \geqslant \sigma_{3} \geqslant \sigma_{1}$. It can be found from Figs. 5-7 that the effects of the deviation from isotropic elasticity and pore fluid compressibility on the band direction angle and the critical hardening modulus are considerable.

(a)

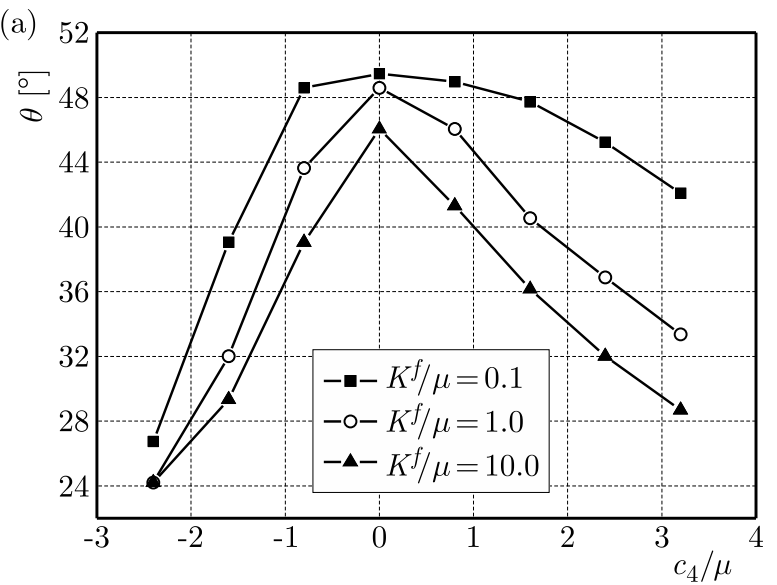

(b)

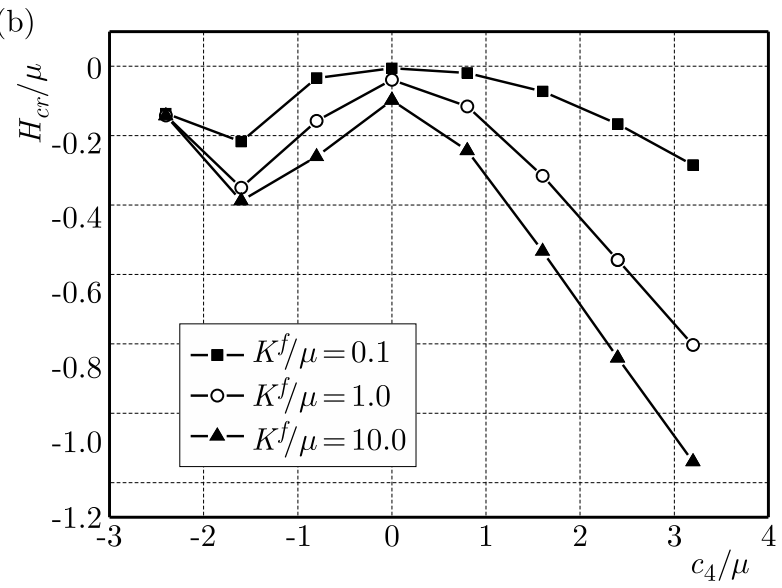

Fig. 5. Relationship between the band direction angle $\theta$ (a) and the critical hardening modulus $H_{c r}^{\prime}$ (b) and parameter $c_{4}$ for different $K^{f}$ for the case $\sigma_{3}^{\prime} \geqslant \sigma_{2}^{\prime} \geqslant \sigma_{1}^{\prime}$

In addition, for both the situations $\sigma_{1}^{\prime} \geqslant \sigma_{3}^{\prime}$ and $\sigma_{3}^{\prime} \geqslant \sigma_{1}^{\prime}$, it can be concluded from the above discussions that the initiation of strain localization for porous media with transversely isotropic elasticity is delayed with the increase of pore fluid compressibility, which is consistent with the conclusions for isotropic porous media drawn by Han and Vardoulakis (1991) and Runesson et al. (1996). For associated plasticity, it is found from the corresponding figures that the values of 
(a)

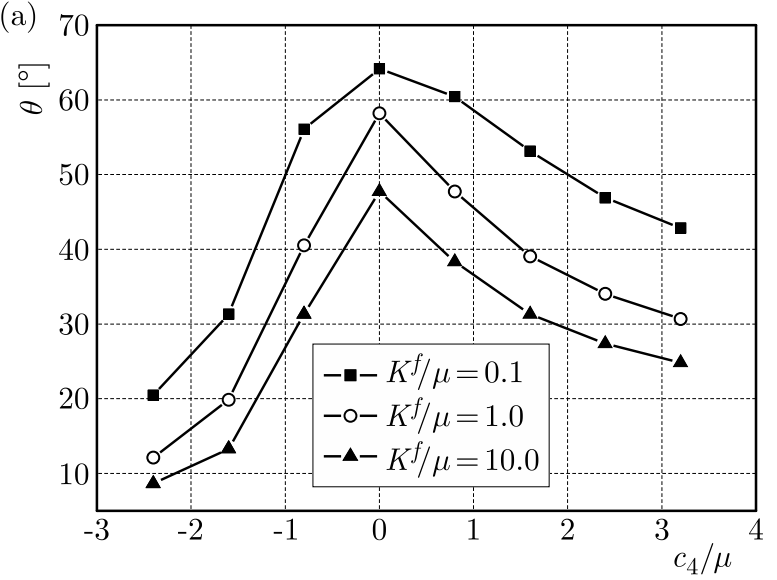

(b)

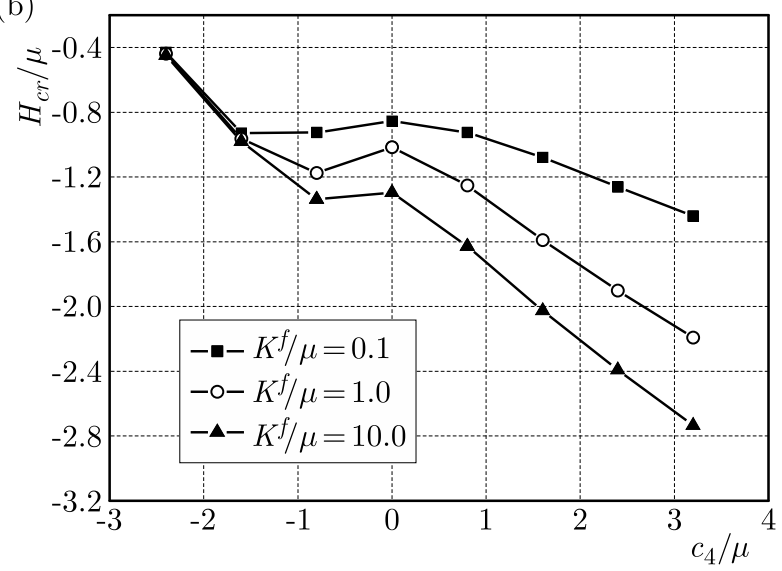

Fig. 6. Relationship between the band direction angle $\theta$ (a) and the critical hardening modulus $H_{c r}^{\prime}$ (b) and parameter $c_{4}$ for different $K^{f}$ for the case $\sigma_{3}^{\prime} \geqslant \sigma_{1}^{\prime} \geqslant \sigma_{2}^{\prime}$

(a)

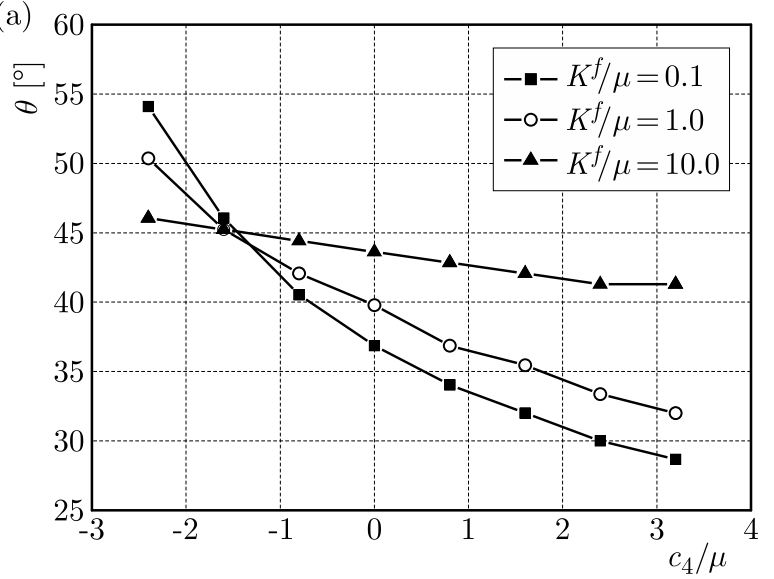

(b)

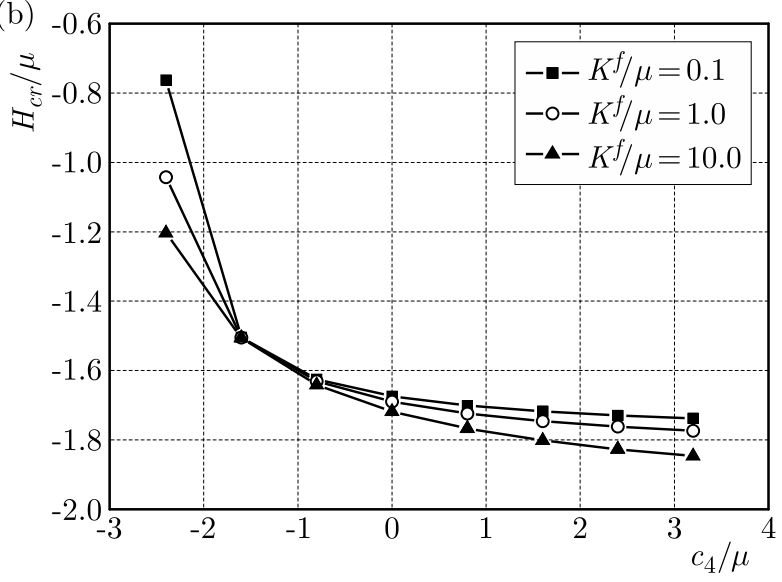

Fig. 7. Relationship between the band direction angle $\theta$ (a) and the critical hardening modulus $H_{c r}^{\prime}$ (b) and parameter $c_{4}$ for different $K^{f}$ for the case $\sigma_{2}^{\prime} \geqslant \sigma_{3}^{\prime} \geqslant \sigma_{1}^{\prime}$

the critical hardening modulus at the onset of strain localization are non-positive, which is in agreement with the point of view proposed by Ottosen and Runesson (1991).

\section{Conclusions}

This study conducts an investigation on the properties of strain localization for elastic-plastic porous media with transversely isotropic elasticity under undrained conditions. Under non-associated plasticity and tri-axial stress states, the conditions for localization of deformation into a band in the incremental response of porous media are derived, in which the effects of the deviation from isotropic elasticity and pore fluid compressibility are taken into account. The explicit expression for the hardening modulus at the onset of strain localization are obtained.

With reference to the Mohr-Coulomb yield criterion, the effects of deviation from isotropic elasticity and pore fluid compressibility in the formulation of strain localization for the case of plane strain are discussed. It turns out that the properties of strain localization are dependent upon the deviation from isotropic elasticity and pore fluid compressibility. The deviation from isotropic elasticity and pore fluid compressibility have great impacts on the direction angle of localized band initiation and the corresponding critical hardening modulus. In general, a larger value of the compression modulus of pore fluid leads to a smaller value of the critical hardening 
modulus at the initiation of strain localization. In other words, the onset of strain localization for elastic-plastic porous media with transversely isotropic elasticity is delayed with an increase in the pore fluid compressibility. The influence of the pore fluid compressibility on the direction angle of localized band is related to the magnitude of deviation from isotropic elasticity.

Acknowledgments

This work has been supported by National Natural Science Foundation of China (NSFC) (No. 11172265).

\section{References}

1. Alyavuz B., Gultop T., 2009, Weak shock waves and shear bands in compressible, inextensible thermoelastic solids, Archive of Applied Mechanics, 79, 1145-1161

2. Bigoni D., LoREt B., 1999, Effects of elastic anisotropy on strain localization and flutter instability in plastic solids, Journal of the Mechanics and Physics of Solids, 47, 1409-1436

3. Gao Z.W., Zhaо J.D., 2013, Strain localization and fabric evolution in sand, International Journal of Solids and Structures, 50, 3634-3648

4. Han C., Vardoulakis I., 1991, Plane strain compression experiments on water-saturated finegrained sand, Geotechnique, 16, 49-78

5. Longere P., Dragon A., 2007, Adiabatic heat evaluation for dynamic plastic localization, Journal of Theoretical and Applied Mechanics, 45, 203-223

6. Loret B., Rizzi E., 1997, Qualitative analysis of strain localization. Part II: Transversely isotropic elasticity and plasticity, International Journal of Plasticity, 13, 501-519

7. Mandel J., 1962, Ondes plastiques dans un milieu indéfini à trios dimensions, Journal de Mécanique I, 1, 3-30

8. Mandel J., 1964, Conditions de Stabilité et Postulat de Drucker, [In:] Kravtchenko J., Sirieys P.M. (Eds.), Rheology and Soil Mechanics, Springer, Berlin, 58-68

9. Nielsin K., Schreyer H.L., 1993, Bifurcations in elastic-plastic materials, International Journal of Solids and Structures, 30, 521-544

10. Ottosen N.S., Runesson K., 1991, Properties of discontinuous bifurcation solutions in elastoplasticity, International Journal of Solids and Structures, 27, 401-421

11. Rice J.R., 1976, The localization of plastic deformation, [In:] Loiter W.T. (Ed.), Proceedings of 14 th IUTAM Congress, Delft, Amsterdam: North Holland, 207-220

12. Rizzi E., Loret B., 1997, Qualitative analysis of strain localization. Part I: Transversely isotropic elasticity and isotropic plasticity, International Journal of Plasticity, 13, 461-499

13. Rizzi E, Loret B., 1999, Strain localization in fluid-saturated anisotropic elastic-plastic porous media, International Journal of Engineering Science, 37, 235-251

14. Rudnicki J.W., 1977, The effect of stress-induced anisotropy on a model of brittle rock failure as localization of deformation, [In:] Wang F.D., Clark G.B. (Eds.), Energy Resources and Excavation Technology, Proceedings of the 18th US Symposium on Rock Mechanics, Keystone, Colorado, 1-8

15. Rudnicki J.W., 2002, Conditions for compaction and shear bands in a transversely isotropic material, International Journal of Solids and Structures, 39, 3741-3756

16. Runesson K., Peric D., Sture S., 1996, Effect of pore fluid compressibility on localization in elastic-plastic porous solids under undrained conditions, International Journal of Solids and Structures, 33, 1501-1518

17. Thomas T.Y., 1961, Plastic Flow and Fracture in Solids, Academic Press, New York 
18. Zhang H.W., Schrefler B.A., 2001, Uniqueness and localization analysis of elastic-plastic saturated porous media, International Journal for Numerical and Analytical Methods Geomechanics, 25, 29-48

19. Zhang H.W., Zhou L., Schrefler B.A., 2005, Material instabilities of anisotropic saturated multiphase porous media, European Journal of Mechanics A/Solids, 24, 713-727

20. Zhang Y.Q., HaO H., Yu M.H., 2002, Effect of porosity on the properties of strain localization in porous media under undrained conditions, International Journal of Solids and Structures, 39, 1817-1831

21. ZHANG Y.Q., Lu Y., YU M.H., 2003, Investigation of strain localization in elastoplastic materials with transversely isotropic elasticity, International Journal of Mechanical Sciences, 45, 217-233

Manuscript received September 19, 2015; accepted for print July 5, 2016 